Review

\title{
Deep brain stimulation of the pedunculopontine nucleus modulates subthalamic pathological oscillations
}

\author{
Alessandro Stefani $^{\mathrm{a}, *}$, Laura Clara Grandi ${ }^{\mathrm{b}}$, Salvatore Galati ${ }^{\mathrm{b}, * *}$ \\ a Department of system medicine, UOSD Parkinson, University of Rome "Tor Vergata", Italy \\ ${ }^{\mathrm{b}}$ Department of Movement Disorders, Neurocenter of Southern Switzerland, Ospedale Civico di Lugano, Switzerland
}

\section{A B S T R A C T}

Low frequency deep brain stimulation (DBS) of the pedunculopontine nucleus area (PPNa) has been proposed as a novel surgical target for gait dysfunction in the late stage of Parkinson's disease (PD). Since the mid-2000s, we have shown that intrasurgical delivery of stimulation in the pontine tegmentum affects the firing activity in the subthalamic nucleus (STN), but its effect on STN oscillatory rhythms has not been studied. Neuronal oscillations detected by local field potential (LFPs) have great importance, since they express complex movement-related behavior such as locomotion. Therefore, we examined the effect of three PPNa-DBS stimulation protocols (at 10, 25 and $80 \mathrm{~Hz}$ ) on the STN oscillatory activity of PD patients. We focused on the anti-kinetic beta $(\beta, 15-30 \mathrm{~Hz})$, the pro-kinetic gamma $(\gamma, 60-90 \mathrm{~Hz})$ and "gaitrelated" alpha $(\alpha, 7-12 \mathrm{~Hz})$ bands. We hypothesized that modulation of STN oscillations might have clinical relevance in the PPNa-mediated effects.

PPNa stimulation at 25 and $80 \mathrm{~Hz}$ decreased the power of the STN $\beta$ band by 33.94 and $40.22 \%$, respectively. PPNa-DBS did not affect the other two bands with a tendency to suppress $\alpha$ power, while $\gamma$ oscillation increased.

Our results suggest that the anti-kinetic $\beta$ band is the oscillation most sensitive to PPNa-DBS despite the negligible clinical efficacy on bradykinesia. However, how these changes interact reciprocally with the cortex or are counterbalanced by lower brainstem/spinal pathways remain to be elucidated.

Our observation might turn out to be helpful in new protocols designed with adaptive DBS supporting the addition of PPN implantation in PD patients experiencing declining efficacy of STN-DBS.

\section{Introduction}

The pedunculopontine nucleus area (PPNa) has been proposed as novel target for deep brain stimulation (DBS) in those Parkinson's disease (PD) patients experiencing levodopa resistant axial symptoms such as freezing of gait and postural instability (Moreau et al., 2009; Stefani et al., 2007). Although the DBS mechanisms have not yet been clarified, the clinical benefit from PPN-DBS may be explained by several mechanisms, including modulation of spinal reflexes (Pierantozzi et al., 2008) or interactions with cerebellar nuclei (Vitale et al., 2016).

However, at least part of the described benefits, when present (Thevathasan et al., 2018), may relate to its reciprocal connections to the basal ganglia (BG) and, particularly, to the subthalamic nucleus (STN) (Bevan and Bolam, 1995; Ceravolo et al., 2011; Galati et al., 2008). In this regard, the lesion of the PPN region or the PPNa-DBS at $25 \mathrm{~Hz}$, in a PD rat model obtained by 6-OHDA-induced chronic denervation (Breit et al., 2006), reduced the pathological hyperactivity of the STN. In addition, PPN-DBS was able to decrease the pathological STN neuronal oscillation in the $\beta$ band $(15-30 \mathrm{~Hz})$ as evaluated in experimental models (Alam et al., 2012). An exaggerated $\beta$ band is associated with the 'off' state in both PD patients and rodents (Brown, 2006; Galati et al., 2006; Mallet et al., 2008). Further, clinical amelioration achieved through dopaminergic therapy or conventional DBS targets is associated with a decrease of this frequency band parallel to an increase of $\gamma$ activity (Brown, 2003; Doyle et al., 2005; Eusebio et al., 2011).

Similar to STN, the $\beta$ band has also been detected in the PPNa of PD patients (Weinberger et al., 2008). However, to our knowledge, only the activity in $\alpha$ frequency, expressed more powerfully in the caudal part of the PPNa, was functionally and inversely related to gait impairment and freezing in PD (Thevathasan et al., 2012). Of note, $\alpha$ synchronization has the role to suppress 'task irrelevant' interference and correlates with improved gait performance during PPNa-DBS in PD patients (Androulidakis et al., 2008a, 2008b).

Whether the clinical impact of PPNa-DBS promotes significant modulation of intra-STN oscillations was never established in human PD. Taking advantage of the combined implantation of the two nuclei as performed by our group in the mid-2000s (Mazzone et al., 2005; Stefani et al., 2007), we previously showed that the large majority of STN neurons responded to $25 \mathrm{~Hz}$ PPN-DBS by changing their firing

*Correspondence to: A. Stefani, Department of System Medicine, UOSD Parkinson Center, University of Rome "Tor Vergata", Fondazione Policlinico Tor Vergata viale Oxford 81, Rome 00133, Italy.

** Correspondence to: S. Galati, Neurocenter of Southern Switzerland, Ospedale Civico di Lugano, via Tesserete, 46, 6900 Lugano, Switzerland.

E-mail addresses: stefani@uniroma2.it (A. Stefani), salvatore.galati@eoc.ch (S. Galati). 
activity depending upon the prestimulation firing pattern (Galati et al., 2008). Since neuronal rhythms have become biomarkers for behaviorrelated neural activity, herein, we re-analyzed these neuronal traces in order to detect the content of oscillatory neural behavior in the STN, focusing on the $\alpha, \beta$, and $\gamma$ bands.

\section{Methods}

Eight PD patients underwent stereotactic electrode (Medtronic model 3389, Medtronic Inc., Minneapolis, Minnesota, USA) implantation according to previously reported criteria (Galati et al., 2006, 2008; Mazzone et al., 2005). These participants were the same as those in our previous study (Galati et al., 2008). Of note, they belong, as previously mentioned, to the first phase of PPN surgery (while, in the modern era, the PPN was unilaterally targeted without simultaneous implantation of STN (see, for instance, Mazzone et al., 2016).

The stereotactic coordinates were described elsewhere (Galati et al., 2008). Briefly, related to the line connecting the anterior (CA) and posterior (CP) commissure, the coordinates to STN were: $\mathrm{x}^{1 / 4} 12 \mathrm{~mm}, \mathrm{y}^{1 / 4}$ CA-CP $1 / 2, \mathrm{z}^{1 / 4} 4 \mathrm{~mm}$; whereas coordinates to PPNa were: $\mathrm{x}^{1 / 4} 47 / 9 \mathrm{~mm}$, $\mathrm{y}^{1 / 41-2} \mathrm{~mm}$ below $\mathrm{CP}$ and $\mathrm{z}^{1 / 413}$ (5 mm below the pontomesencephalic line).

The modern experience (as detailed in Mazzone et al., 2014) clarified that stereotactic coordinates to PPNa (re-named as PPTg) are based not simply on standard CA-CP, but upon brain-stem inter-individual differences, corroborated by potentials recorded from lemniscus medialis as each of the four electrode contacts cross it. For further details, please consider the functional anatomy described extensively elsewhere (i.e. Mazzone et al., 2013, 2014); in principle, specific brain stem measures are adopted being the ponto-mesencephalic junction a crucial reference. For example, the sagittal parameters are reconstructed on sagittal pre-operative MRI slices and designated as i) S1, from the plane formed by the top of the midbrain - middle point of the posterior commissure - to the pontomesencephalic junction (PMJ); ii) S2 distance, between the PMJ and the Obex; iii) S1 distance, between the top of the midbrain and the Obex ("brainstem long axis"); IV) SW distance, corresponding to the widths of the pons measured along the fastigial floor line (and additional as available in Mazzone et al., 2013, 2014). Besides, any patient underwent post-operative TC or MRI (exemplary MRI in Suppl. Fig. 1).

Written informed consent was obtained from each patient. The investigation was approved by the local ethics committee. Quadripolar electrodes were stereotactically implanted in both the STN and PPN under local anesthesia in two different surgical sessions. Paolo Mazzone was the neurosurgeon pioneering the procedure (Mazzone et al., 2005). The stereotactic coordinates are described elsewhere (Galati et al., 2008); Mazzone et al., 2013, 2014). Briefly, related to the line connecting the anterior (CA) and posterior (CP) commissure, the coordinates to the STN were $\mathrm{x}^{1 / 4} 12 \mathrm{~mm}, \mathrm{y}^{1 / 4}$ CA-CP $1 / 2, \mathrm{z}^{1 / 4} 4 \mathrm{~mm}$, whereas coordinates to the PPNa were $\mathrm{x}^{1 / 47} / 9 \mathrm{~mm}, \mathrm{y}^{1 / 4} 1-2 \mathrm{~mm}$ below CP and $\mathrm{z}^{1 / 413}$ ( $5 \mathrm{~mm}$ below the pontomesencephalic line).

Microelectrode recordings with high impedance tungsten electrodes (0.5-1.5 MO, FHC) were attempted in all surgeries but were successfully performed in six out of eight patients and single unit recordings were considered suitable for analysis when lasting $>20 \mathrm{~min}$, allowing correlation with different DBS protocols. STN recordings were amplified (ISO-DAM8; World Precision Instruments, Hertfordshire, UK), sampled $(50 \mathrm{kHz})$ on-line, stored into a computer connected to a CED 1401 interface, and analyzed off-line (Spike 2, CED, Cambridge, UK). PPN-DBS was performed by a Medtronic external device Model 3625 (Medtronic Inc.) with the following parameters: pulses consisting of $60 \mu$ s width and 2-3 V (bipolar stimulation, contact 0-1) at 10, 25 and $80 \mathrm{~Hz}$. PPN-DBS lasted at least $600 \mathrm{~s}$ (mean 649 s738).

The frequency content of neurons with oscillatory activity was evaluated by performing a spectral analysis of the continuous waveform obtained by transforming spike trains in a density of spikes in time (sampling rate $1 \mathrm{kHz}$ ). This analysis was performed using conversion software from Spike2, CED (Galati et al., 2009; Levy et al., 2000). The size of the transform used in the fast Fourier transform (FFT) analysis was 512 points, thereby yielding a frequency resolution of $1.95 \mathrm{~Hz}$. We focused on $\alpha(7-12 \mathrm{~Hz}), \beta(13-30 \mathrm{~Hz})$ and $\gamma(60-90 \mathrm{~Hz})$ bands. We collected a total of 6, 3 and 2 recordings from STN during PPN stimulation of 10,25 and $80 \mathrm{~Hz}$, respectively, of 2 patients. We calculated the total power of $\alpha(7-12 \mathrm{~Hz}), \beta(13-30 \mathrm{~Hz})$ and $\gamma(60-90 \mathrm{~Hz})$ band in STN during $30 \mathrm{~s}$ of baseline and $30 \mathrm{~s}$ of successive PPN stimulation. Only patients with a clear recording file to be transformed for oscillatory activity investigation were taken into account for the analysis.

The brief recording protocols discouraged the opportunity to verify UPDRS items and $\backslash$ or cognitive tasks. For each frequency stimulation, indeed, we averaged the $30 \mathrm{~s}$ of baseline and $30 \mathrm{~s}$ of stimulation of all trials, comparing them by means of the nonparametric Wilcoxon Signed Ranks test, since the nonnormally distribution of data subjected to a Kolmogorov-Smirnov test.

Then, we calculate the percentage of power changes in $\alpha, \beta$ and $\gamma$ bands before and during stimulation. Clinical assessment concerning the evaluation of rigidity and the finger tapping sequence has been reported previously (Galati et al., 2008).

\section{Results}

A $30 \mathrm{~s}$ of PPN-DBS at $10 \mathrm{~Hz}$ determined a slight decrease of the STN $\beta$ band equal to $9.97 \%$ with a certain trend toward significance $(p=.102$; Fig. 1).

At 25 and $80 \mathrm{~Hz}$, the $\beta$ power significantly decreased up to $33.94 \%$ $(p=.005$; Fig. 1$)$ and to $40.22 \%(p=.002$ Fig. 1$)$, respectively.

All the stimulation protocols decreased the $\alpha$ power equal to $24.37 \%, 10.69 \%$ and $34.32 \%$ at 10,25 and $80 \mathrm{~Hz}$, respectively, with a marginally significant trend $(10 \mathrm{~Hz} p=.106 ; 25 \mathrm{~Hz} p=.229 ; 80 \mathrm{~Hz}$ $p=.781$; Fig. 1).

$\gamma$ power slightly decreased during the two lower stimulation frequencies, whereas it tended to increase with the $80 \mathrm{~Hz}$ stimulation $(-1.30 \%$ and $-6.18 \%$ and $+11.32 \% ; 10 \mathrm{~Hz} p=.943 ; 25 \mathrm{~Hz} p=.975$; $80 \mathrm{~Hz} p=.147$; Fig. 1).

\section{Discussion}

In the present study, we observed that PPNa-DBS had an impact on oscillatory behavior of STN neurons. Interestingly, PPNa stimulation at 25 and $80 \mathrm{~Hz}$ produced a significant decrease of $\beta$ frequency. A clear trend toward reduction of the $\beta$ band was observed in the $10 \mathrm{~Hz}$ stimulation. The significant dampening of the STN $\beta$ band, as detected at 25 and $80 \mathrm{~Hz}$ PPNa-DBS, is in line with the traditional BG literature (for a review, see Stefani et al., 2018). The relation between $\beta$ activity and the parkinsonian state has been widely demonstrated in PD patients and reproduced in animal models affecting different areas of the BG critically involved in PD such as the STN (Başar and Güntekin, 2008; Eusebio et al., 2009; Little and Brown, 2014; Litvak et al., 2011; Oswal et al., 2013).

Consistently, dopaminergic therapies and STN-DBS attenuated pathological neuronal oscillations in $\beta$ frequencies within the STN with a strict correlation with cardinal signs of Parkinsonian syndrome such as bradykinesia and rigidity (Bronte-Stewart et al., 2009; Brown, 2006; Kuhn et al., 2008). Similarly, it has been demonstrated that PPN-DBS in a PD animal model determined the decrease of the $\beta$ oscillations of the STN (Alam et al., 2012).

Clinical studies have shown that PPN-DBS at low $20-25 \mathrm{~Hz}$ has positive effects on gait and posture deficits of PD patients (Moro et al., 2010; Plaha and Gill, 2005), whereas higher frequency rates have been reported to be not effective or even detrimental to motor performance. Nevertheless, the electrophysiological consequences of both $25 \mathrm{~Hz}$ and $80 \mathrm{~Hz}$, as detected here, should be examined more carefully in a larger series and, critically, in different settings (i.e., exploring chronic 


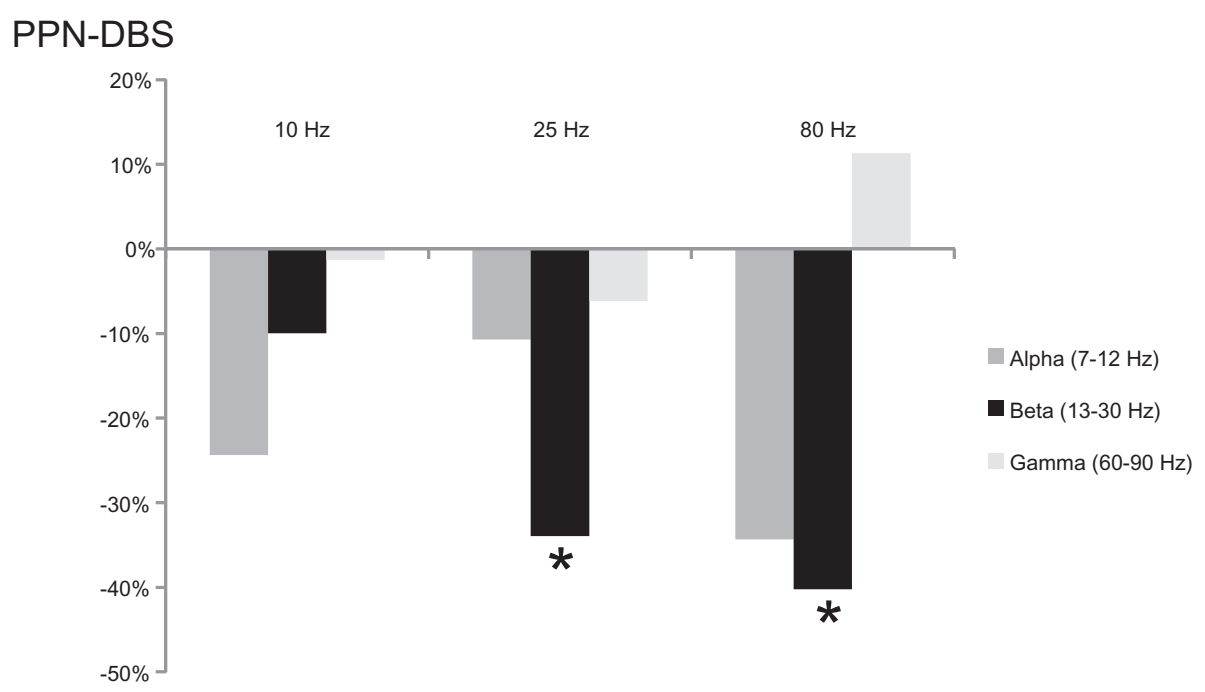

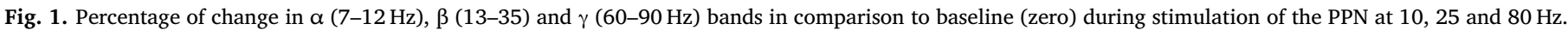

effects). In fact, higher frequencies in the $\gamma$ frequency range are required to optimally induce locomotion reflecting the predominance of this band in the activity of the single PPN neurons recorded in vitro (Garcia-Rill et al., 2016). Herein, the $\gamma$ band in the STN was negligibly affected by PPNa-DBS at 10 and $25 \mathrm{~Hz}$, whereas it was partially engaged at $80 \mathrm{~Hz}$.

The stronger impact of higher frequency stimulation on the STN $\beta$ band suggests that the positive clinical outcome on axial signs obtained by PPNa-DBS at low frequency could be linked to mechanisms not involving descending pathways independent of the STN. At higher stimulation protocols, an overdrive of bottom pathways antagonizes the clinical benefit of $\beta$ band reduction within the STN.

While the $\beta$ band exerts a prominent role in motor control and has been associated with anti-kinetic properties (Zhang et al., 2008), the $\alpha$ band has a role in different kinds of cognitive and attentive functions (Klimesch, 2012; Klimesch et al., 2007), facilitating processing of task relevant information in specific brain areas.

During high frequency stimulation of the STN, there was significant attenuation of both $\alpha$ and $\beta$ band LFP power (Bronte-Stewart et al., 2009), while at lower frequencies $(60 \mathrm{~Hz})$ a significant increase has been observed in the $\alpha$ band (8-12 Hz; (Blumenfeld et al., 2017)). Indeed, in freely moving PD patients a clinically beneficial $60 \mathrm{~Hz}$ DBS amplified $\alpha$ and low- $\beta(11-15 \mathrm{~Hz}, P=.007)$ and attenuated high- $\beta$ power $(19-27 \mathrm{~Hz}, P<.001)$ in the STN, suggesting an implicit "decoupling of the cortico-STN hyperdirect pathway"(Blumenfeld et al., 2017). The partial incongruence of our results with these data ( $\alpha$ band not significantly changed during PPNa-stimulation) could be attributed to profound differences in circuitry properties of PD patients manifesting dramatically different clinical patterns (our cohort did in fact include subjects whose freezing could be negligible). Moreover, the difference may be due to a methodological issue, since we have studied how the stimulation of an area (PPNa) influenced the oscillatory activity of another region (STN) instead of intra-STN stimulation.

The analysis of motor performance under PPN-STIM (as reported in (Galati et al., 2008)) demonstrated the lack of significant changes, in terms of acute impact on rigidity and on finger tapping sequence. Here, we documented change of the STN beta band. Our results could appear contradictory. However, the decrease of the abnormal STN beta band during a brief PPN stimulation sequence may not be automatically translated into improving of motor activities (while chronic PPN_STIM, in combination with submaximal LD, proved to confer a synergistic significant amelioration of hypokinetic signs (Stefani et al., 2007).

In addition, the STN belongs to a rather complex circuitry. Indeed, the final motor phenotype is determined by interactions of different areas of the circuit, including the DBS-mediated antidromic modulation of the STN-cortical pathway; our data are limited to the mere interaction between PPNa and the STN and, by default, do not explore more complex interactions with cortex.

Overall, here we demonstrated for the first time the effect of PPNaDBS on STN oscillation patterns, a critical area involved in PD. We posited a long time ago that the PPNa could be considered a novel target for PD treatment by mean of DBS. Even if it was not possible to analyze patient motor scores for a prolonged time due to surgical concerns, we suggest that PPNa stimulation might provide an additional tool to influence amelioration of hypokinetic signs, since abnormal $\beta$ oscillations are associated with motor dysfunction.

A recent review (Thevathasan et al., 2018) suggested that despite the use of different methods/trajectories hampering a global estimation of clinical results and limiting the clinical application of PPN surgery, PPN DBS improved gait freezing, reduced falls with a variable degree of improvement, and influenced the non-motor symptom profile in selected patients. It is difficult to define whether the specific PD patients who benefit more from PPN-STIM indeed feature a larger remodulation of $\beta$ discharge.

Unfortunately, our original approach (Stefani et al., 2007), based on complex 4 lead neurosurgery, were not replicated consistently. Instead, the monolateral implantation of PPNa has been tested by different groups with conflicting results (Mazzone et al., 2016; Thevathasan et al., 2018).

Our present findings raise the possibility that the add-on implantation of PPNa might represent an explorable technique in PD patients previously implanted bilaterally in the STN, the benefits of which progressively decline in correlation with axial deficit or cognitive decline. This might be adequately tested in protocols of adaptive DBS ensuring real-time monitoring of electrode LFPs.

Supplementary data to this article can be found online at https:// doi.org/10.1016/j.nbd.2018.11.006.

\section{Conflict of interest}

The authors declare do not have any financial arrangements or connections pertinent to the submitted manuscript.

\section{Financial disclosure}

None. 


\section{Acknowledgments}

The authors are particularly grateful to Ninfa Amato for critical reading. SG received support from Parkinson Schweiz and Fondazione ticinese per le malattie neurodegerative per l'adulto e l'anziano.

\section{References}

Alam, M., Heissler, H.E., Schwabe, K., Krauss, J.K., 2012. Deep brain stimulation of the pedunculopontine tegmental nucleus modulates neuronal hyperactivity and enhanced beta oscillatory activity of the subthalamic nucleus in the rat 6-hydroxydopamine model. Exp. Neurol. 233, 233-242. https://doi.org/10.1016/j. expneurol.2011.10.006.

Androulidakis, A.G., Khan, S., Litvak, V., Pleydell-Pearce, C.W., Brown, P., Gill, S.S., 2008a. Local field potential recordings from the pedunculopontine nucleus in a Parkinsonian patient. Neuroreport 19. https://doi.org/10.1097/WNR. 0b013e3282f2e2d1.

Androulidakis, A.G., Mazzone, P., Litvak, V., Penny, W., Dileone, M., Gaynor, L.M.F.D., Tisch, S., Di Lazzaro, V., Brown, P., 2008b. Oscillatory activity in the pedunculopontine area of patients with Parkinson's disease. Exp. Neurol. 211. https://doi.org/ 10.1016/j.expneurol.2008.01.002.

Başar, E., Güntekin, B., 2008. A review of brain oscillations in cognitive disorders and the role of neurotransmitters. Brain Res. 1235, 172-193. https://doi.org/10.1016/j. brainres.2008.06.103.

Bevan, M.D., Bolam, J.P., 1995. Cholinergic, GABAergic, and glutamate-enriched inputs from the mesopontine tegmentum to the subthalamic nucleus in the rat. J. Neurosci. $15,7105-7120$.

Blumenfeld, Z., Koop, M.M., Prieto, T.E., Shreve, L.A., Velisar, A., Quinn, E.J., Trager, M.H., Brontë-Stewart, H., 2017. Sixty-hertz stimulation improves bradykinesia and amplifies subthalamic low-frequency oscillations. Mov. Disord. Off. J. Mov. Disord. Soc. 32, 80-88. https://doi.org/10.1002/mds.26837.

Breit, S., Lessmann, L., Unterbrink, D., Popa, R.C., Gasser, T., Schulz, J.B., 2006. Lesion of the pedunculopontine nucleus reverses hyperactivity of the subthalamic nucleus and substantia nigra pars reticulata in a 6-hydroxydopamine rat model. Eur. J. Neurosci. 24, 2275-2282. https://doi.org/10.1111/j.1460-9568.2006.05106.x.

Bronte-Stewart, H., Barberini, C., Koop, M.M., Hill, B.C., Henderson, J.M., Wingeier, B., 2009. The STN beta-band profile in Parkinson's disease is stationary and shows prolonged attenuation after deep brain stimulation. Exp. Neurol. 215, 20-28. https:// doi.org/10.1016/j.expneurol.2008.09.008.

Brown, P., 2003. Oscillatory nature of human basal ganglia activity: relationship to the pathophysiology of Parkinson's disease. Mov. Disord. Off. J. Mov. Disord. Soc. 18, 357-363. https://doi.org/10.1002/mds.10358.

Brown, P., 2006. Bad oscillations in Parkinson's disease. J. Neural Transm. In: Suppl.

Ceravolo, R., Brusa, L., Galati, S., Volterrani, D., Peppe, A., Siciliano, G., Pierantozzi, M., Moschella, V., Bonuccelli, U., Stanzione, P., Stefani, A., 2011. Low frequency stimulation of the nucleus tegmenti pedunculopontini increases cortical metabolism in parkinsonian patients. Eur. J. Neurol. Off. J. Eur. Fed. Neurol. Soc. 18, 842-849. https://doi.org/10.1111/j.1468-1331.2010.03254.x.

Doyle, L.M.F., Kuhn, A.A., Hariz, M., Kupsch, A., Schneider, G.-H., Brown, P., 2005. Levodopa-induced modulation of subthalamic beta oscillations during self-paced movements in patients with Parkinson's disease. Eur. J. Neurosci. 21, 1403-1412. https://doi.org/10.1111/j.1460-9568.2005.03969.x.

Eusebio, A., Pogosyan, A., Wang, S., Averbeck, B., Gaynor, L.D., Cantiniaux, S., Witjas, T., Limousin, P., Azulay, J.-P., Brown, P., 2009. Resonance in subthalamo-cortical circuits in Parkinson's disease. Brain J. Neurol. 132, 2139-2150. https://doi.org/10. 1093/brain/awp079.

Eusebio, A., Thevathasan, W., Doyle Gaynor, L., Pogosyan, A., Bye, E., Foltynie, T., Zrinzo, L., Ashkan, K., Aziz, T., Brown, P., 2011. Deep brain stimulation can suppress pathological synchronisation in parkinsonian patients. J. Neurol. Neurosurg. Psychiatry 82, 569-573. https://doi.org/10.1136/jnnp.2010.217489.

Galati, S., Mazzone, P., Fedele, E., Pisani, A., Peppe, A., Pierantozzi, M., Brusa, L., Tropepi, D., Moschella, V., Raiteri, M., Stanzione, P., Bernardi, G., Stefani, A., 2006. Biochemical and electrophysiological changes of substantia nigra pars reticulata driven by subthalamic stimulation in patients with Parkinson's disease. Eur. J. Neurosci. 23, 2923-2928. https://doi.org/10.1111/j.1460-9568.2006.04816.x.

Galati, S., Scarnati, E., Mazzone, P., Stanzione, P., Stefani, A., 2008. Deep brain stimulation promotes excitation and inhibition in subthalamic nucleus in Parkinson's disease. Neuroreport 19, 661-666. https://doi.org/10.1097/WNR.0b013e3282fb78af.

Galati, S., Stanzione, P., D'Angelo, V., Fedele, E., Marzetti, F., Sancesario, G., Procopio, T., Stefani, A., 2009. The pharmacological blockade of medial forebrain bundle induces an acute pathological synchronization of the cortico-subthalamic nucleus-globus pallidus pathway. J. Physiol. 587, 4405-4423. https://doi.org/10.1113/jphysiol. 2009.172759.

Garcia-Rill, E., Luster, B., D'Onofrio, S., Mahaffey, S., Bisagno, V., Urbano, F.J., 2016. Implications of gamma band activity in the pedunculopontine nucleus. J. Neural Transm. Vienna Austria 1996 (123), 655-665. https://doi.org/10.1007/s00702-0151485-2.

Klimesch, W., 2012. $\alpha$-Band oscillations, attention, and controlled access to stored information. Trends Cogn. Sci. 16, 606-617. https://doi.org/10.1016/j.tics.2012.10. 007.

Klimesch, W., Sauseng, P., Hanslmayr, S., 2007. EEG alpha oscillations: the inhibitiontiming hypothesis. Brain Res. Rev. 53, 63-88. https://doi.org/10.1016/j.brainresrev 2006.06.003.
Kuhn, A.A., Kempf, F., Brucke, C., Gaynor Doyle, L., Martinez-Torres, I., Pogosyan, A., Trottenberg, T., Kupsch, A., Schneider, G.-H., Hariz, M.I., Vandenberghe, W., Nuttin, B., Brown, P., 2008. High-frequency stimulation of the subthalamic nucleus suppresses oscillatory beta activity in patients with Parkinson's disease in parallel with improvement in motor performance. J. Neurosci. 28, 6165-6173. https://doi.org/10. 1523/JNEUROSCI.0282-08.2008.

Levy, R., Hutchison, W.D., Lozano, A.M., Dostrovsky, J.O., 2000. High-frequency synchronization of neuronal activity in the subthalamic nucleus of parkinsonian patients with limb tremor. J. Neurosci. 20, 7766-7775.

Little, S., Brown, P., 2014. The functional role of beta oscillations in Parkinson's disease. Parkinsonism Relat. Disord. 20 (Suppl. 1), S44-S48. https://doi.org/10.1016/S13538020(13)70013-0.

Litvak, V., Jha, A., Eusebio, A., Oostenveld, R., Foltynie, T., Limousin, P., Zrinzo, L., Hariz, M.I., Friston, K., Brown, P., 2011. Resting oscillatory cortico-subthalamic connectivity in patients with Parkinson's disease. Brain J. Neurol. 134, 359-374. https://doi.org/10.1093/brain/awq332.

Mallet, N., Pogosyan, A., Marton, L.F., Bolam, J.P., Brown, P., Magill, P.J., 2008. Parkinsonian beta oscillations in the external globus pallidus and their relationship with subthalamic nucleus activity. J. Neurosci. 28, 14245-14258. https://doi.org/10. 1523/JNEUROSCI.4199-08.2008.

Mazzone, P., Lozano, A., Stanzione, P., Galati, S., Scarnati, E., Peppe, A., Stefani, A., 2005. Implantation of human pedunculopontine nucleus: a safe and clinically relevant target in Parkinson's disease. Neuroreport 16, 1877-1881.

Mazzone, P., Sposato, S., Insola, A., Scarnati, E., 2013. The clinical effects of deep brain stimulation of the pedunculopontine tegmental nucleus in movement disorders may not be related to the anatomical target, leads location, and setup of electrical stimulation. Neurosurgery 73 (5), 894-906.

Mazzone, P., Paoloni, M., Mangone, M., Santilli, V., Insola, A., Fini, M., Scarnati, E., 2014. Unilateral deep brain stimulation of the pedunculopontine tegmental nucleus in idiopathic Parkinson's disease: effects on gait initiation and performance. Gait Posture 40, 357-362.

Mazzone, P., Vilela Filho, O., Viselli, F., Insola, A., Sposato, S., Vitale, F., Scarnati, E., 2016. Our first decade of experience in deep brain stimulation of the brainstem: elucidating the mechanism of action of stimulation of the ventrolateral pontine tegmentum. J. Neural Transm. Vienna Austria 1996. https://doi.org/10.1007/ s00702-016-1518-5.

Moreau, C., Defebvre, L., Devos, D., Marchetti, F., Destée, A., Stefani, A., Peppe, A., 2009. STN versus PPN-DBS for alleviating freezing of gait: toward a frequency modulation approach? Mov. Disord. Off. J. Mov. Disord. Soc. 24, 2164-2166. https://doi.org/10. $1002 /$ mds. 22743.

Moro, E., Hamani, C., Poon, Y.-Y., Al-Khairallah, T., Dostrovsky, J.O., Hutchison, W.D., Lozano, A.M., 2010. Unilateral pedunculopontine stimulation improves falls in Parkinson's disease. Brain J. Neurol. 133, 215-224. https://doi.org/10.1093/brain/ awp261.

Oswal, A., Brown, P., Litvak, V., 2013. Synchronized neural oscillations and the pathophysiology of Parkinson's disease. Curr. Opin. Neurol. 26, 662-670. https://doi.org/ 10.1097/WCO.0000000000000034.

Pierantozzi, M., Palmieri, M.G., Galati, S., Stanzione, P., Peppe, A., Tropepi, D., Brusa, L., Pisani, A., Moschella, V., Marciani, M.G., Mazzone, P., Stefani, A., 2008. Pedunculopontine nucleus deep brain stimulation changes spinal cord excitability in Parkinson's disease patients. J. Neural Transm (Vienna) 115, 731-735. https://doi. org/10.1007/s00702-007-0001-8. Epub 2008 Jan 15, PubMed PMID: 18196202.

Plaha, P., Gill, S.S., 2005. Bilateral deep brain stimulation of the pedunculopontine nucleus for Parkinson's disease. Neuroreport 16, 1883-1887.

Stefani, A., Lozano, A.M., Peppe, A., Stanzione, P., Galati, S., Tropepi, D., Pierantozzi, M., Brusa, L., Scarnati, E., Mazzone, P., 2007. Bilateral deep brain stimulation of the pedunculopontine and subthalamic nuclei in severe Parkinson's disease. Brain J. Neurol. 130, 1596-1607. https://doi.org/10.1093/brain/awl346.

Stefani, A., Cerroni, R., Mazzone, P., Liguori, C., Di Giovanni, G., Pierantozzi, M., Galati, S., 2018. Mechanisms of action underlying the efficacy of deep brain stimulation of the subthalamic nucleus in Parkinson's disease: central role of disease severity. Eur. J. Neurosci. https://doi.org/10.1111/ejn.14088.

Thevathasan, W., Pogosyan, A., Hyam, J.A., Jenkinson, N., Foltynie, T., Limousin, P., Bogdanovic, M., Zrinzo, L., Green, A.L., Aziz, T.Z., Brown, P., 2012. Alpha oscillations in the pedunculopontine nucleus correlate with gait performance in parkinsonism. Brain J. Neurol. 135, 148-160. https://doi.org/10.1093/brain/awr315.

Thevathasan, W., Debu, B., Aziz, T., Bloem, B.R., Blahak, C., Butson, C., Czernecki, V., Foltynie, T., Fraix, V., Grabli, D., Joint, C., Lozano, A.M., Okun, M.S., Ostrem, J., Pavese, N., Schrader, C., Tai, C.-H., Krauss, J.K., Moro, E., Movement Disorders Society PPN DBS Working Groupin collaboration with the World Society for Stereotactic and Functional Neurosurgery, 2018. Pedunculopontine nucleus deep brain stimulation in Parkinson's disease: a clinical review. Mov. Disord. Off. J. Mov. Disord. Soc. 33, 10-20. https://doi.org/10.1002/mds.27098.

Vitale, F., Mattei, C., Capozzo, A., Pietrantoni, I., Mazzone, P., Scarnati, E., 2016 Mar 11. Cholinergic excitation from the pedunculopontine tegmental nucleus to the dentate nucleus in the rat. Neuroscience. 317, 12-22. https://doi.org/10.1016/j. neuroscience.2015.12.055. Epub 2016 Jan 4, PubMed PMID: 26762800.

Weinberger, M., Hamani, C., Hutchison, W.D., Moro, E., Lozano, A.M., Dostrovsky, J.O., 2008. Pedunculopontine nucleus microelectrode recordings in movement disorder patients. Exp. Brain Res. 188, 165-174. https://doi.org/10.1007/s00221-0081349-1.

Zhang, Y., Wang, T., Ma, A., Zhou, X., Gui, J., Wan, H., Shi, R., Huang, C., Grace, A.A., Huang, C.L.-H., Trump, D., Zhang, H., Zimmer, T., Lei, M., 2008. Correlations between clinical and physiological consequences of the novel mutation R878C in a highly conserved pore residue in the cardiac Na + channel. Acta Physiol. Oxf. Engl. 194, 311-323. https://doi.org/10.1111/j.1748-1716.2008.01883.x. 